\title{
Reaction times and attention in Parkinson's disease
}

\author{
C A BLOXHAM,* D J DICK, $\dagger$ M MOORE $\ddagger$ \\ From the Departments of Pathology* and Neurology, $\uparrow$ Newcastle General Hospital and the Department of \\ Medical Statistics, $\ddagger$ University of Newcastle, Newcastle upon Tyne, UK
}

SUMMARY Ten patients with Parkinson's disease performed a simple reaction time task in which, on hearing a tone, they pressed a button with the left thumb. In the first experiment tones sometimes occurred unannounced and at other times were preceded (by between 0 and $3200 \mathrm{~ms}$ ) by a warning signal. The second experiment was identical to the first except that the subject had simultaneously to perform a simple continuous task with his right hand. Patients had slower reaction times than controls under all circumstances. In general, however, the effect of a warning signal and the effect of a second task were the same for both groups. In the control group the effect of a warning signal depended on whether or not a second task was being performed. Specifically, the advantage of a warning signal for reaction time was lost after long intervals ( $>200 \mathrm{~ms})$ when a second task was being performed. Parkinson's disease patients lost this advantage even when they were not performing a second task. Animal studies have suggested that dopamine deficiency results in an increase in neural "noise" in the basal ganglia. The behavioural consequences of this may be that Parkinson's disease patients always perform as if they were carrying out another task at the sam® time. In contrast, their ability to benefit from a warning signal and to allocate attentional resources are unimpaired.

One of the cardinal symiptoms of Parkinson's disease is difficulty in initiating and performing movements, and there have been several recent attempts to understand the fundamental nature of this deficit. In particular, simple and choice reaction time experiments have suggested that one of the underlying reasons for this bradykinesia may be a difficulty in setting up motor programmes prior to action. ${ }^{12}$ In these studies subjects may or may not be warned which hand will be required to respond to a subsequent signal. Patients with Parkinson's disease appear to respond as quickly as controls when no preparatory signal is given, but their reaction times do not significantly decrease if a prior warning "left" or "right" is provided. They seem to be slow in priming the motor programme appropriate to the intended movements. This concept invites the question as to whether Parkinsonian patients also have difficulty in acquiring and maintaining a motor "set" if given a temporal warning only, with no additional requirement to

Address for reprint requests: Dr C A Bloxham, Department of Pathology, Newcastle General Hospital, Newcastle upon Tyne NE4 6BA, UK.

Received 4 July 1986 and in revised form 23 October 1986. Accepted 29 October 1986 select any particular response. One aim of the present study was to explore this possibility. The experimeno involved the response by a designated hand to an imperative signal which occurred at a variable inter val after a warning. Earlier work has shown that under these conditions normal subjects will decrease their reaction time by up to $50 \mathrm{~ms}$ if allowed a warning-response interval in the range of 300 to $500 \mathrm{~ms}^{34}$

The other main aspect of this study was to determine the attentional requirements of people with Parkinson's disease during the performance of simple movements. Patients often complain that they require a conscious effort to complete straightforward daily activities which had been performed easily and automatically before they became ill. ${ }^{5}$ In some cases the difficulty may be compounded by rigidity and tremor but the clinical picture suggests that an abnormally high sustained concentration is required for the movements themselves. Indeed, this may be one of the causes of the common symptoms of tiredness and fatigue in Parkinson's disease.

A frequently employed method of investigating the allocation of attention is the use of experiments on dual task performance. For example, the simple reaction time to an imperative signal may be measured when the latter is presented at various times during 
the performance of a second task. Such studies have suggested that attentional requirements are greater at the beginning and end of a movement than in the middle. ${ }^{6}$ For this investigation, no such discrimination was attempted, and the global resource requirements of a simple oscillatory movement of an arm were estimated by employing the same reaction time paradigm described above during the performance of such a movement. In this way, the effects of a minor load on the preparation time for a motor programme could be evaluated.

Finally, although the clinical response to levodopa has been well documented, there have been few studies of the effects of medication on reaction time in Parkinson's disease. Although complicated by a wide qualitative and quantitative variation in response to drugs many patients will improve at between 45 minutes and 2 hours following a dose of levodopa. ${ }^{7}$ An attempt was therefore made to perform the above experiments under conditions both of partial drug withdrawal and optimum treatment using this information as a guideline.

\section{Method}

\section{Task I}

Subjects were required to respond to a sound by pressing the "FIRE" button of a joystick as quickly as possible with the thumb of the non-dominant hand. Successive trials occurred at random intervals of between 3.5 and 10.0 seconds following the subject's previous response. The imperative signal was either unwarned or else preceded by the word "READY" on the VDU at periods of $100,200,800$ or $3200 \mathrm{~ms}$ before the sound. The latter two times had an additional random component of between $+20 \%$ and $-20 \%$ of the stated value. The five possible combinations occurred in random order until a defined and equal number of each had been realised as described below. Reaction times for each trial were recorded by a $B B C$ computer to the nearest $10 \mathrm{~ms}$.

\section{Task 2}

The second experiment consisted of a repeat of Task 1 during the performance of a simple paced oscillatory movement by the dominant hand. The subject was required to move a small hexagonal "sprite" on the monitor between two rectangular boxes measuring 5.0 by $1.4 \mathrm{~cm}$ in area and $7.0 \mathrm{~cm}$ apart. The position of the sprite was controlled by the dominant hand with a joystick contained within the same handset as the "fire-button" and movement of the sprite was restricted to the horizontal direction. The handset was held by the non-dominant hand with the thumb on the "fire-button" as in the previous task. The subject was instructed to maintain a continuous and steady rhythm by "bouncing" the sprite between the boxes in time with a bleep emitted by the computer at a constant frequency of $1 \mathrm{~Hz}$. (The pitch of this bleep was made clearly distinct from the "imperative" noise of the first task.) Reaction times were recorded as for the first task. Subjects were informed that although this task was naturally more complex than the first, the object was again to see how quickly they could react to the imperative signal. This response was therefore to be given priority during the experiment.

The secondary task was not tightly constrained in that the targets were wide and the position of the sprite was undefined during movement. This policy was adopted so that the task would be easy enough to be performed by people with a debilitating motor illness and yet provide sufficient difficulty to permit the allocation of resources by the patients to be assessed. However, the lack of constraints for the task also meant that the performance of the secondary task could not be monitored exactly. It was therefore accepted that slight differences might exist between subjects in the exact form of their movements and no aspect of the secondary task was measured. Instead, it was ensured during the practice session that all subjects could make a smooth, continuous and regular movement of the sprite and subjects were observed during the experiment to confirm that this pattern was maintained.

\section{Performance of task}

Patients were asked to attend having missed their last dose of levodopa (due from 3.25 to 16 (mean 9.5) hours before the experiments were performed). They were then assessed for disability on a modified Webster rating scale and given a Blessed mini-mental state examination. The modification entailed the use of an ordinal scale (0-3) rating of each arm independently for bradykinesia, rigidity, and tremor. The other parts of the Webster scale were used in the standard way and the final score represented the sum of all components. They were then allowed a practice session of 25 trials of Task 1 to ensure that it could be performed adequately and so that errors and anticipation would be minimised. After a short rest (approximately 2 minutes) 50 trials of Task 1 were repeated and the data stored for analysis. A 25 trial practice session for Task 2 was then given and the necessity of smooth movement synchronous with the pacing bleeps emphasised to the subject. Again after a short rest a further 50 trials of Task 2 were performed.

The patients were then allowed their missed medication dose and a repeat Webster rating scale was performed after 1 hour by the same investigator. The above experiments were then repeated in the same order. Control subjects were tested after the same interval in similar fashion.

\section{Subjects}

Ten patients with Parkinson's disease were tested. There were seven men and three women aged between 38 and 68 years (mean age $60 \cdot 2$ ) with a history of symptoms for at least 2 years (table). No subject showed evidence of cognitive impairment and all scored at least 36 out of 37 on the Blessed mini-mental state test. All were right-handed. A possible eleventh subject was unable to perform the tasks due to severe tremor.

The control group consisted of 10 volunteers (seven men and three women) from the technical staff of the hospital and the local community. They were aged between 40 and 67 years (mean age 58.7). All but one were right-handed. Informed consent was obtained from every subject.

\section{Analysis of performance}

Reaction times of less than $150 \mathrm{~ms}$ or greater than 1.5 times the current mean were excluded from the analysis. Within 
Table Clinical status of the Parkinson's disease group

\begin{tabular}{|c|c|c|c|c|c|c|}
\hline Subject & $\operatorname{Sex}$ & Age (yr) & Duration (yr) & Drugs & Ist Rating & 2nd Rating \\
\hline $\begin{array}{r}1 \\
2 \\
3 \\
4 \\
5 \\
6 \\
7 \\
8 \\
9 \\
10\end{array}$ & $\begin{array}{l}\mathbf{F} \\
\mathbf{M} \\
\mathbf{M} \\
\mathbf{M} \\
\mathbf{F} \\
\mathbf{M} \\
\mathbf{F} \\
\mathbf{M} \\
\mathbf{M} \\
\mathbf{M}\end{array}$ & $\begin{array}{l}64 \\
66 \\
38 \\
51 \\
57 \\
67 \\
68 \\
59 \\
67 \\
65\end{array}$ & $\begin{array}{r}2 \\
10 \\
15 \\
5 \\
12 \\
15 \\
5 \\
10 \\
14 \\
7\end{array}$ & $\begin{array}{l}\text { Madopar } \\
\text { Madopar } \\
\text { Sinemet } \\
\text { Sinemet } \\
\text { Sinemet } \\
\text { Madopar } \\
\text { Madopar } \\
\text { Madopar } \\
\text { Sinemet } \\
\text { Sinemet }\end{array}$ & $\begin{array}{l}13 \\
24 \\
11 \\
11 \\
16 \\
20 \\
10 \\
10 \\
15 \\
11\end{array}$ & $\begin{array}{r}5 \\
16 \\
13 \\
10 \\
8 \\
16 \\
5 \\
7 \\
10 \\
9\end{array}$ \\
\hline
\end{tabular}

and between group comparisons were made using analysis of variance with adjustment for repeated measures using a BMDP biomedical package. The statistical significance

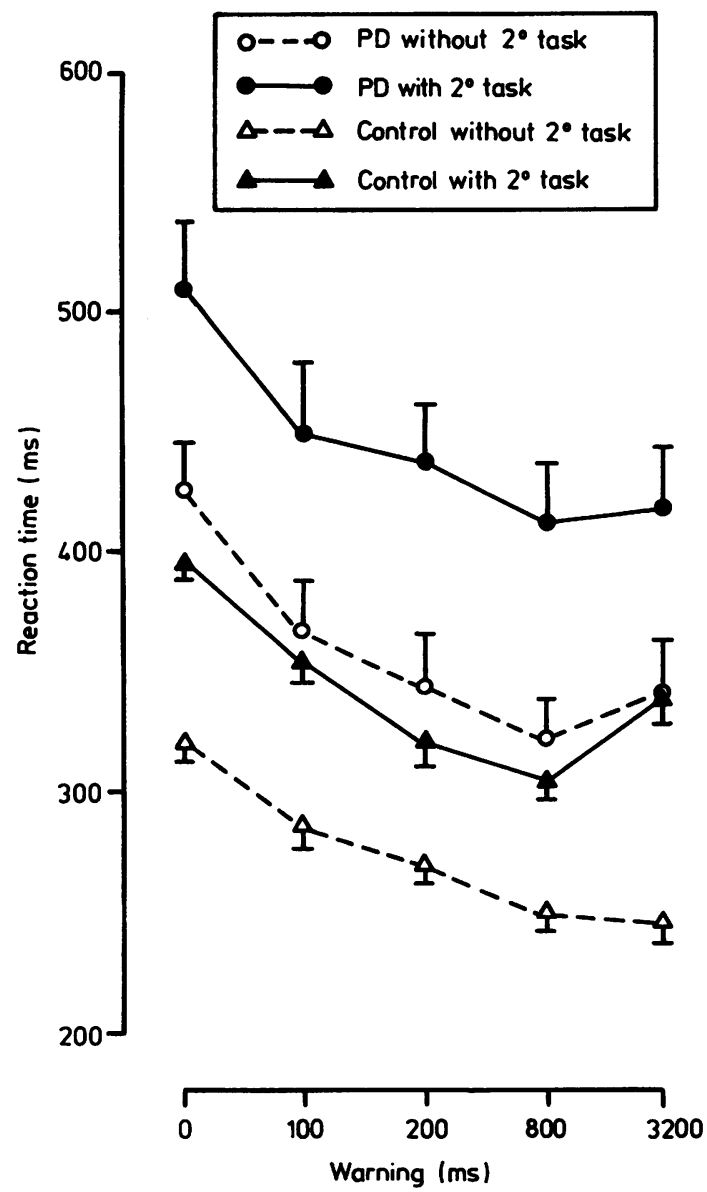

Fig 1 The effects of length of forewarning period and performance of secondary task on reaction times of Parkinsonian subjects who are levodopa deficient. (Bars indicate SEM.) levels are given with the Greenhouse-Geiser correction where this is appropriate.

\section{Results}

Figure 1 shows the results of both groups with and without the secondary tasks for the first session. Figure 2 shows the data obtained during the second session.

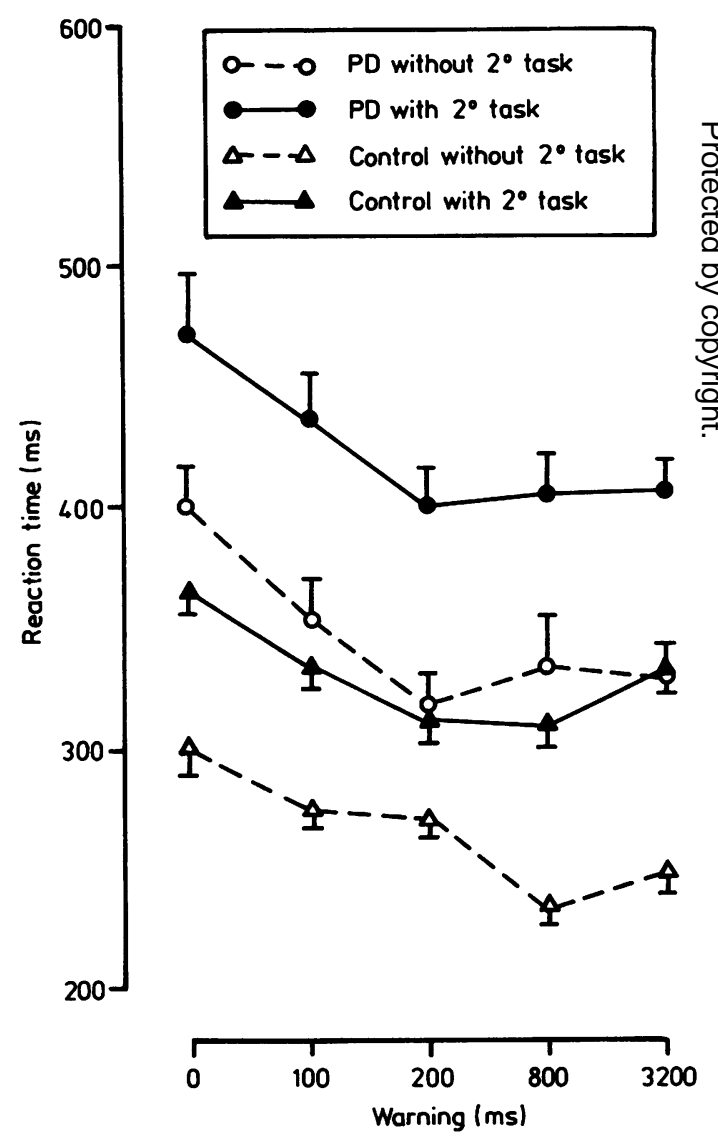

Fig 2 As for fig l but Parkinsonian subjects are at one hour following levodopa administration. (Bars indicate SEM.) 

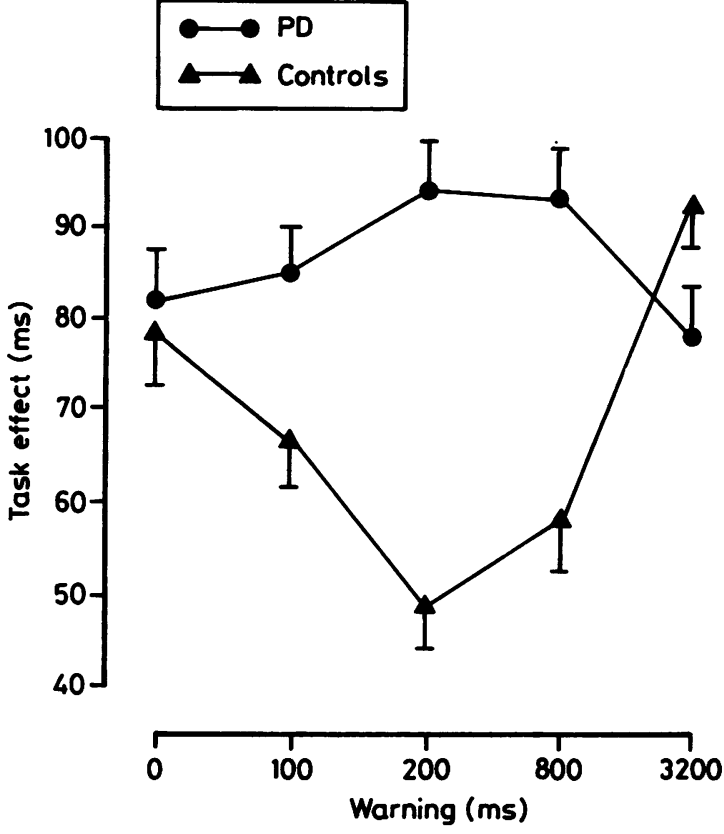

Fig 3 The gain in reaction time due to the performance of a secondary task as a function of the leng th of the forewarning period. (Bars indicate SEM.)

It is clear that at each warning interval during both sessions the Parkinsonian group was much slower than the control. Nevertheless, there was a marked beneficial effect of warning for both groups with and without the secondary task. However, this benefit was not equal under all conditions and there was a significant interaction involving task $\times$ warning $\times$ group $(F(4,72)=4.33, p<0.005)$. Consequently, the effects of task, warning and group cannot be analysed separately. The interaction was evident in the non-parallel nature of the four curves of fig 1 but was demonstrated more clearly when the differences between task and no-task reaction times were plotted at each warning interval for the two groups (fig 3 ). The Parkinson's disease curve is much flatter than that for the controls which is U-shaped curve with a minimum at $200 \mathrm{~ms}$. If Task 2 (dual task performance) is analysed separately there is no interaction between warning time and group $(\mathrm{F}(4,72)=$ $1 \cdot 37, p=0 \cdot 26$ ). Also, analysis of the patient group alone shows no interaction between task and warning time $(F(4,36)=0.38, p=0.74)$. This implies that both curves for the patient group and the control group with task are all parallel and that the difference in slopes of the two curves in fig 3 is principally due to the relative flatness of the control group performing reaction times without another task. The other main feature of fig 3 is that there is no statistically significant difference between the values with no warning for the two groups.

At one hour following medication all but one patient showed a decrease in their rating scores and all but one reported a small subjective improvement (table). There was a small global improvement in all reaction times of both groups in the second session but this was not large enough to attain statistical significance $(\mathrm{F}(1,18)=2 \cdot 23, \mathrm{p}>0 \cdot 15)$.

\section{Discussion}

Previous reaction-time experiments have shown that if patients with Parkinson's disease are given no information regarding which hand is required for a motor response their reaction times are not significantly different from those of controls. ${ }^{12}$ In contrast, whilst control subjects are able to decrease their reaction times if prewarned for laterality no such improvement is obtained by people with Parkinsonism. The present study supports these findings: one hand only was used for the key-press and therefore the response side was always known (the experiment corresponds to the simple reaction time paradigm used in previous studies). Both before and after the hour interval control times were significantly faster than those of Parkinson's patients for all warning-imperative intervals, irrespective of the presence or absence of the secondary task. This suggests that in Parkinson's disease information regarding which muscles are to be employed in a subsequent task cannot be used as efficiently to prime the relevant motor programme prior to its execution.

However, there is a clear benefit for the patients if a temporal warning only is supplied and this appears equally early for both groups $(100 \mathrm{~ms})$. (The possibility remains that a difference in benefit may occur at a warning between 0 and $100 \mathrm{~ms}$ as this interval was not examined in the present experiment.) A similar dissociation between these two types of task was observed by Rabbitt and Vyas ${ }^{4}$ who showed that whereas older people are less able than the young to use information regarding which of two possible responses will be required, their ability to gain advantage from temporal warnings alone remained unimpaired. The fact that there is also an age-related loss of dopamine in the basal ganglia of people without clinical Parkinson's disease ${ }^{8}$ supports the notion that their difficulty with simple reaction times is a specific motor deficit related to reduced activity of the ascending dopamine pathways, and not a feature of brain disease or aging in itself. These observations also suggest that the neuronal mechanisms responsible for alerting subjects are not dopamine dependent and may relate to pathways not directly involving the basal ganglia at all. 
Although all four curves indicate that both groups under both conditions benefit from a temporal warning, the interaction between warning and task indicates that the gains do not have the same magnitude at each warning interval. This can be seen by inspection of the graphs. The curves for the patients both with and without task and that for controls with task have a similar, almost parallel, curvilinear appearance. However, the curve for the control group without a task is much different, being both flatter and straighter than the other three curves. This contrast is emphasised by examining the difference between corresponding values of reaction times at each warning interval for both groups (fig 3). That for the Parkinson's disease group is much more nearly horizontal than the control curve which shows a marked dip to a minimum at $200 \mathrm{~ms}$ before rising again to its previous value. The shape of the control curve is partly explained by the relatively rapid response at $200 \mathrm{~ms}$ by controls engaged in a task. The other main contribution is the shallowness of the control curve without task. One interpretation of the similarity in shape of the upper three curves in fig 1 (and thus the relative flatness of the Parkinsonian curve in fig 3 ) is that patients with this illness behave in some ways as if they were constantly performing a motor task even though apparently at rest. This is unlikely to be due to concomitant tremor as this symptom was minimal or absent in many of the patients studied. Furthermore, unless suffering from the side-effects of drugs, patients do not display other abnormal movements such as dyskinesias: poverty of movement is the hallmark of Parkinson's disease.

A possible resolution of this apparent paradox is suggested by recent electrophysiological investigations in primates which have also given evidence of the importance of the basal ganglia in the control of normal movement. Direct recording has shown that for the majority of responsive neurons in the putamen their responses relate to movement whereas those in the head of the caudate show activity mainly during the preparation and initiation periods. ${ }^{9}$ Furthermore, direct iontophoretic application of dopamine decreases the spontaneous firing rate of most of the neurons in these two areas and also those in the adjacent prefrontal cortex. An attractive interpretation of these results is that the basal ganglia are increasing the signal to noise ratio of information processing within this system and that a dopamine deficiency results in reduced transmission through the striatum of at least some forms of cortical information. ${ }^{9}$ The motor consequences of this dysfunction may be the prolonged reaction times observed in this and other studies. ${ }^{12}$ Conversely, the simultaneous performance by a control subject of a secondary task could be described in terms of unwanted "noise" with respect to the required motor response to the auditory signal. Although the "noise" now has a different origin, the reaction times under these circumstances approach those of Parkinsonian patients who are not performing a secondary task and the shapes of the two reaction time curves are similar. Thus the results of these psychological experiments are consistent with the concepts derived from animal neurophysiological studies.

If no warning is given the increase in reaction time when a secondary task is performed is the same for patients and controls. This indicates that there are no extra attentional requirements for Parkinsonian subjects who engage in a motor activity, contrary to expectations based on clinical observations. However, there are several reasons to resist such a conclusion on the basis of this study. Firstly, the task was very simple: a more complicated task may bring to light the use of greater resources by patients during movement. Secondly, there may be an increased attentional requirement at the beginning of a movement only and this was not specifically assessed. Thirdly, since the Parkinsonian curve is higher, for whatever reason, than the controls, then the absolute increase in reaction time may not reflect the corre sponding attentional requirements in the same way as it does for the controls. Further experiments are needed to settle these issues.

The insignificant difference in reaction times obtained before and after treatment is initially surs prising but might be explained by the fact that the clinical improvement was variable as judged by the Webster rating scale or by subjective impression. may be necessary to study patients in unequivocat $\overrightarrow{0}$ ON or OFF states before the relationship between the reaction times and clinical status can be adequately assessed.

There is a wide range of motor disturbances in Parkinson's disease which although taken together present a well defined clinical picture are not easy to understand conceptually. The results of psychomotor experiments have added to the complexity by revealing abnormalities at several levels in the motor hierarchy and also within more than one motor system (for example both peripheral and eye movement control). A recurring theme, however, is the attempt to describe at least some aspects of the motor deficit in terms of information processing models. In this context it seems important to know which psychological structures are preserved as well as those that are impaired. We suggest that one of the consequences of the dopamine deficiency in Parkinson's disease is a loss of definition of a specific motor programme which results in a decreased ability to activate it selectively from those available in the repertoire. Nevertheless patients maintain their ability to 
benefit from alerting signals and their allocation of attentional resources to the performance of simple tasks may be normal.

We thank Dr CD Frith for helpful comments on this manuscript and for encouragement. We are grateful to Dr JB Foster for allowing us to study patients under his care and the Whickham Health Centre, Newcastle for access to patients and for their hospitality.

\section{References}

I Bloxham CA, Mindel TA, Frith CD. Initiation and execution of predictable and unpredictable movements in Parkinson's disease. Brain 1984;107:371-84.

2 Evarts EV, Tervainen H, Calne DB. Reaction times in Parkinson's disease. Brain 1981;104:167-86.

3 Posner MI. The Chronometric Analysis of Mind. New Jersey: L Erlbaum Associates, 1979.
4 Rabbitt PMA, Vyas S. Age and the rate of preparation for signals and for responses. Exp Brain Res 1982; Suppl 5:216-22.

5 Marsden CD. The mysterious motor function of the basal ganglia: The Robert Wartenberg lecture. Neurology 1982;32:14-39.

6 Klein RM. Attention and movement. In: Stelmach GM, ed. Motor Control; Issues and Trends. New York: Academic Press, 1976

7 Muenter MD, Tyce GM. L-DOPA therapy of Parkinson's disease: plasma concentration, therapeutic response and side effects. Mayo Clin Proc 1971;46:231-9.

8 Carlsson A, Winblad B. Influence of age and time interval between death and autopsy on dop;amine and 3-methoxytyramine levels in human basal ganglia. J Neurol Transm 1976;38:271-6.

9 Rolls ET, Thorpe SJ, Boytim M, Szabo I, Perrott DI. Responses of striatal neurones in the behaving monkey: 3. Effects of iontophoretically applied dopamine on normal responsiveness. Neuroscience 1984;12: 1201-12. 